Background and aims Multimodal haemodynamic monitoring has an important role in PICU, because that can aid the intensivist to perform the management of children with acute circulatory failure.

The aim is to improve the interest of haemodynamic management with multimodal parameters to answer to the 3 most commonly asked questions: Complete clinical diagnosis, guide therapeutics, and repeat measures for evaluation.

Methods In this prospective study, between January 2012 and April 2014, the assessment of haemodynamic was obtained progressively by NIBP, TTE, and estimated continuous cardiac output (esCCO), and/or Oesophageal Doppler, an/or pleth variability index (PVI) for each patient with ACF.

Results On a total of 33 patients with ACF, all patients was treated:

- Volume expansion in 31 patients "fluid responsiveness based on the respiratory variation aortic flow peak velocity $\Delta$ Vpeak ao, SV, and inferior vena cava, and/or PVI, FTc, $\Delta$ Vpeak by OD .

- 29 responder ( $\Delta \mathrm{SV} \geq 10 \%$ by TTE, esCCO and/or OD).

- 2 non responder $(\Delta \mathrm{SV}<10 \%)$.

- Norepinephrine was introduced in 14 patients (objective MAP and or PPC for SPTBI), 2 of them had a profound vasoplegia « DAP $<40 \mathrm{mmHg}$.

- Dobutamine was introduced in 2 patients with LVEF $<45 \%$ (TTE).

Conclusion MHM allows optimisation of systemic haemodynamic: assessment of $\mathrm{CO}$, blood volume status, vascular resistance and contractility.

\section{PO-0330 HEALTHCARE-ASSOCIATED INFECTIONS IN A PAEDIATRIC INTENSIVE CARE UNIT}

J Extreia, M Rodrigues, C Camilo, L Boto, J Rios, F Abecasis, M Vieira. Pediatric Intensive Care Unit Pediatric Department, University Hospital of Santa Maria, Lisbon, Portugal

\subsection{6/archdischild-2014-307384.979}

Introduction Healthcare-associated infections (HAI) are a common cause of higher morbidity, mortality and longer stay in PICU.

Objective Characterisation of HAI: tracheobronchitis, pneumonia, bloodstream infection (BSI) and urinary tract infection (UTI) in our PICU, for a 12 month period (2013).

Methods Retrospective review of clinical data from patients admitted $>48 \mathrm{~h}$, using a modified patient-based HELICS protocol. HAI was defined according to the Centre for Disease Control.

Results From a total of 450 admissions, 233 patients were included. Mean age was 6,7 years (0-18), mean length of stay was 6,6 days (3-67), and the majority had antibiotic on admission $(87 \%)$. Seventy one patients $(31 \%)$ were mechanically ventilated, $41 \%$ had CVC and 33\% a urinary catheter.

Fifteen children had a total of 21 HAI (9\%): 16 respiratory infections - 13 pneumonias and 3 tracheobronchitis (19,8 and 4,6/1000 days of ventilation, respectively), 2 primary BSI - one related to CVC (1,4/1000 days of CVC) and 3 UTI (2,9/1000 days of urinary catheter). The most common pathogens were Pseudomonas aeruginosa and Enterobacter cloacae in respiratory infections. Staphylococcus hominis and Candida parapsilosis were identified in BSI and Escherichia coli, Enterococcus faecalis, Candida parapsilosis in UTI. Mean length of stay was 26,9 days in the HAI group versus 5,2 days in non HAI group. There was no HAI related mortality.

Discussion The incidence of HAI was similar to other European Units. We found a higher rate of respiratory infections than that of a previous study in our PICU, emphasising the importance of monitoring and preventive measures.

\section{PO-0331 THE EFFECTS OF INCREASED AWARENESS ON MEDICATION ERROR DISCLOSURES}

L Fernandez-Restrepo, J Ruiz, MS Son, R Taylor. Pediatric Critical Care, The University of Texas Health Science at San Antonio, San Antonio, USA

\subsection{6/archdischild-2014-307384.980}

Background Communicating medication errors is a crucial part of patient care. Children are exposed up to three times the rate of potentially dangerous adverse drug events. A previous evaluation of disclosure of medication errors identified barriers in communicating these errors.

Methods Two presentations on medication errors and how to improve their communication with patient/family were presented to the PICU team. A Medication Error Data Entry Form was used to collect the number and type of medication errors (only type $\mathrm{C}$ through I require immediate notification to the $\mathrm{MD}$ ). Communication to family was documented in a separate form.

Results Thirty-four medication errors were recorded over a 4 month period ( 2 months before and 2 months after education). Fifty-three percent were type A errors (circumstances or events that have the capacity to cause error) while the remaining were type C (an error occurred that reached the patient but did not cause patient harm) (Table 1).

\section{Abstract P0-0331 Table 1}

\begin{tabular}{|l|c|ccc|}
\hline Group & \multicolumn{2}{c|}{ Type A Error Type C Error } & MD Notified & Family Notified \\
\hline Before Education & $46.7 \%$ & $22.2 \%$ & $100.0 \%$ & $50 \%$ \\
\hline After Education & $20.0 \%$ & $75.0 \%$ & $91.7 \%$ & $31 \%$ \\
\hline
\end{tabular}

While the fellows did not participate in the communication of errors to patient/family before education, they did in $60 \%$ of the notifications afterwards. The two barriers to communication were "family was not available" (43\%) and "error did not cause side effects" (57\%).

Conclusion This study demonstrates that despite the effort to increase awareness of medication errors disclosure there was not an improvement in communicating of medication errors to the patient/family. A more systematic and aggressive approach to education on communication may be required to properly address and improve the disclosure of medication errors.

\section{PO-0332 BARRIERS TO DISCLOSURE OF MEDICATION ERRORS}

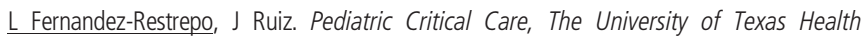
Science at San Antonio, San Antonio, USA

\subsection{6/archdischild-2014-307384.981}

Background Medication administration error is the most frequent error in paediatrics and one of the leading causes of death.Adverse event reporting is critical to improving patient 
safety but challenging in our field, and not without barriers. The purpose of this analysis was to determine how often medications errors get communicated to patients and/or families and to identify the barriers perceived by healthcare providers to disclose these errors.

Methods A survey was distributed to the critical care team. The questions were answered anonymously, with the only identifier being their position in the critical care team.

Results A 76\% response was obtained, eight attendings (44.4\%), 5 nurses-RNs (27.8\%), 3 nurse practitioners- NPs (16.7\%), and 3 fellows (16.7\%) returned the survey. The group that 'always' reported communication of medicine errors was the attendings (42\%), followed by RNs (40\%), NPs (25\%), and the fellows $(0 \%)$ (Figure 1). The most often perceived obstacle to communicating was family not being available (Figure 2).

Conclusion This analysis demonstrated that communication of medication errors does not happen consistently. In addition, the most common obstacle identified was the absence of family when the event occurs. This is most likely a challenge that is more unique to the paediatric population. The culture of open communication is critical in creating a safer medical environment; therefore, it is a skill that must be implemented into the medical education.

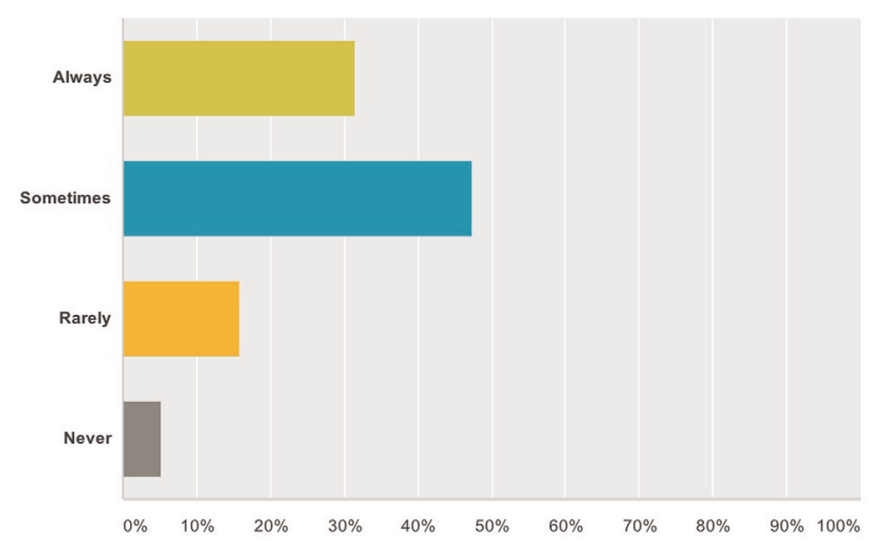

Abstract P0-0332 Figure 1 Frequency of communication of medication errors to the patient/family

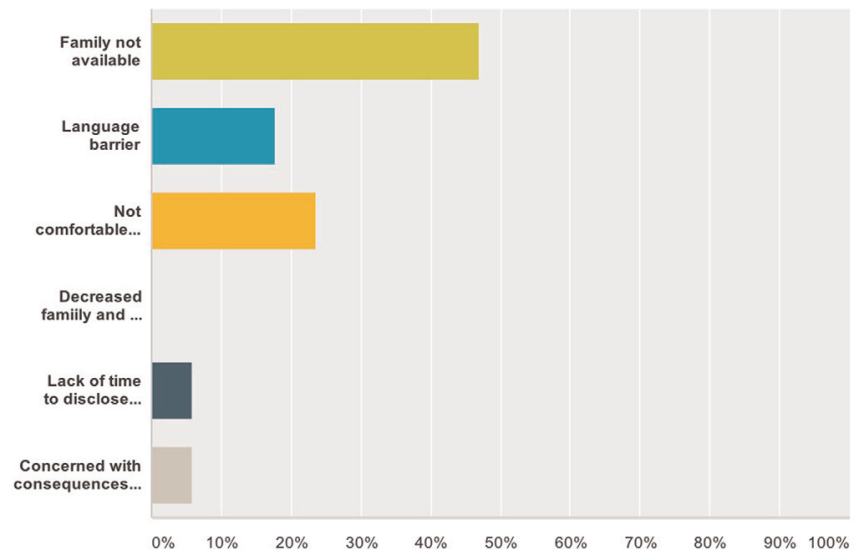

Abstract P0-0332 Figure 2 Barriers encountered in communicating medication errors to a patient/family

\section{PO-0333 MUSCLE THICKNESS ULTRASONOGRAPHY IN CRITICALLY ILL CHILDREN: ANALYSIS OF ACCURACY}

${ }^{1} \mathrm{~T}$ Fivez, ${ }^{1} \mathrm{~A}$ Hendrickx, ${ }^{2} \mathrm{~F}$ Claus, ${ }^{1} \mathrm{~L}$ Desmet, ${ }^{1} \mathrm{D}$ Vlasselaers, $1,3 \mathrm{~T}$ Van Herpe, ${ }^{1} \mathrm{G}$ Van den Berghe, 'D Mesotten. ' $\mathrm{KU}$ Leuven, Dept Intensive Care Medicine, Leuven, Belgium; ${ }^{2}$ OLVZ-Aalst, Radiology Unit, Belgium; ${ }^{3} \mathrm{KU}$ Leuven, Dept Electrical EngineeringESAT-SCD/iMinds Future Health

\subsection{6/archdischild-2014-307384.982}

Background and aims Critical illness results in muscle wasting, typically within the first week of admission. However, its quantification is difficult. Ultrasonographical evaluation has already been tested in adult patients. Here we aim to assess of the accuracy of this methodology in critically ill children.

Methods Two independent investigators made ultrasonographical assessments of muscle thickness. A linear array commercial real time ultrasound scanner (Vivid S6) was used with a $12-\mathrm{MHz}$ transducer. The transducer was placed perpendicularly to the long axis of the tight on three fifths of the distance from the anterior superior iliac spine to the superior patellar border. An excess of contact gel was applied to minimise image distortion. During the first week of critical illness we hypothesised a reduction in muscle size of $30 \%$ based on literature of critically ill adult patients.

Results A group of 43 patients (newborns to 4-years old children) were included in the study. The average muscle thickness was $1.67 \mathrm{~cm}$ (SD: $0.52 \mathrm{~cm}$ ). Hence 30\% reduction would equal $0.50 \mathrm{~cm}$. The intra-observer variability, as expressed by the limits of agreement $( \pm 1.96 \mathrm{SD}$, containing $95 \%$ of the samples for normally distributed samples), was $0.61 \mathrm{~cm}$ for operator 1 and $0.83 \mathrm{~cm}$ for operator 2 . Both distributions are visualised in a Bland-Altman plot in Figure 1. Combining both operator data revealed an observer variability of $0.72 \mathrm{~cm}$. The median absolute inter-observer variability was $0.085 \mathrm{~cm}$ [IQR $0.040-0.20 \mathrm{~cm}$ ]. Conclusions Although the inter-observer variability among the two operators is acceptable, the intra-observer variability may be too large with respect to the limited expected muscle reduction. Therefore, ultrasonographical analyses of muscle wasting need more standardisation for use in critically ill children.

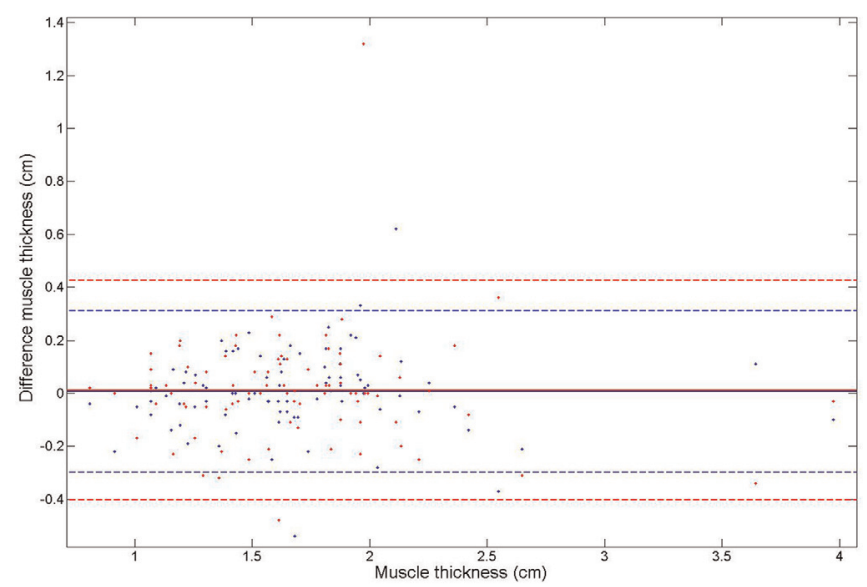

Abstract P0-0333 Figure 1 